This is the post peer-review accepted manuscript of:

Salvaro, Mattia, Victor Kartsch, Simone Benatti, Michela Milano, and Luca Benini. "Towards a novel HMI paradigm based on mixed EEG and indoor localization platforms." In 2017 New Generation of CAS (NGCAS), pp. 217-220. IEEE, 2017. The published version is available online at:

https://doi.org/10.1109/NGCAS.2017.74

(C) 2017 IEEE. Personal use of this material is permitted. Permission from IEEE must be obtained for all other uses, in any current or future media, including reprinting/republishing this material for advertising or promotional purposes, creating new collective works, for resale or redistribution to servers or lists, or reuse of any copyrighted component of this work in other works. 


\title{
Towards a novel HMI paradigm based on mixed EEG and indoor localization platforms
}

\author{
M. Salvaro ${ }^{\dagger}$, V. Kartsch*, S. Benatti*, M. Milano ${ }^{\dagger}$, L.Benini* ${ }^{*}$ \\ *DEI, University of Bologna, Italy. Email: \{victorjavier.kartsch, simone.benatti, luca.benini\}@unibo.it \\ ${ }^{\dagger}$ DISI, Università di Bologna, Italy. Email: \{mattia.salvaro2, michela.milano@ \}unibo.it \\ ${ }^{\ddagger}$ Integrated System Laboratory, ETHZ, Zurich, Switzerland. Email: lbenini@iis.ee.ethz.ch
}

\begin{abstract}
Location Based Services (LBS) have been gaining a great deal of attention thanks to their capability to enhance mobile services with location awareness. While outdoor localization is almost universally achieved via Global Positioning System (GPS), indoor localization is still challenging and a general solution is yet to be found. In a vision where wearable devices are taking over smartphones' leading role as gateway to the cyber world, new paradigms of interactive Human Machine Interfaces (iHMI) are arising. Among others, one of the most intriguing alternative iHMI is based on decoding the brain signals. Combining EEG activity data and indoor localization could dramatically improve the pervasiveness of the interaction between human, devices and environment. For these reasons, we propose a portable Hardware-Software platform that acquires brain EEG signals using a dedicated board along with position information from a cloud service. The positive results of the preliminary analysis successfully show the correlation between EEG signal and motion.

Understanding that this is one of the first intents to merge these two sources of information, we intend to share publicly the ever-growing dataset to allow other researchers to investigate better the interaction between subjects and environments, and to lay the foundation of new paradigms in HMI.
\end{abstract}

\section{INTRODUCTION}

Geolocation is the enabling technique for a wide spectrum of Location-Based Services (LBS) involving, for instance, navigation, transport, tourism, entertainment, healthcare and augmented reality applications. While outdoor localization is mostly solved by Global Positioning System (GPS) technology, indoor localization is still an open issue due to heterogeneity of indoor environments and impracticality of a common approach for all single cases [1]. In fact, GPS is an unreliable technology for indoor positioning, since its signal is not strong enough to be correctly received inside buildings.

Several approaches for indoor localization have been proposed. Some of them exploit existing communication infrastructures like WiFi [2] and FM radio signals [3], others rely on ad-hoc infrastructures like ZigBee [4], Bluetooth [5], Ultra Wide Band (UWB) [6] and Radio Frequency Identification (RFID) [7]. Other systems do not need any kind of specific infrastructure, since they compute the device position using embedded sensors like magnetometer [8], accelerometer, and gyroscope. Furthermore, several hybrid approaches have been proposed, based on complementary technologies like inertial sensors and WiFi [9] or Bluetooth and WiFi [10].

Together with indoor positioning, research on new paradigms for mobile interactive Human-Machine Interfaces (iHMI) has lately gained interest. Following this trend, in a close future, the iHMI could potentially rely only on wearable devices for an integrated and intuitive system that extracts information exclusively from biomarkers [11].
Particularly, EEG signals carry sensitive information about the user's intentions, mood or physiological state. Previous studies have demonstrated that brain activity can be spontaneously triggered by environmental stimuli (colors, visual patterns, aroma, sound, etc.) [12] [13] and the triggered emotions [14]. Similarly, evoked potential response can be extracted when a particular visual or auditory stimuli is presented [15]. Thus, merging geolocation with EEG response can serve as a tool to understand the brain activity during daily tasks, that may empower the efficiency of future iHMI to interpret the user's intentions and functional state of mind.

As mentioned above, many research efforts are focused in the fields of EEG signal processing and indoor positioning, separately. However, to the best of our knowledge, only few contributions aimed at merging the two information sources. In [16], the authors propose a Brain-Machine Interface (BMI) for controlling a wheelchair, where the BMI is based on the P300 evoked potential, triggered though the oddball paradigm technique. The authors also implemented an optical-based tracking system to achieve autonomous navigation. In [17], the author proposes a cloud based solution for patients localization and monitoring in terms of voice pathology detection. In this solution, EEG signal is acquired through a deeply embedded system attached to the outer surface of the patients' vocal fold, while localization is performed via GPS and WiFi.

Differently from the aforementioned works, our main goals are to create a Hardware-Software platform to bind the EEG response with the position of the subject, to understand the challenges on the acquisition of EEG signals (artifacts, external noise and hardware constrains) in real environments, and to study the correlations between the brain activity and the subject's location or movements. To enhance the access to the study of the existing correlations, we intend to make the obtained dataset publicly available.

To achieve our goals, we designed a system to acquire EEG signals from a moving subject while tracking his/her position inside a building. Subject tracking is performed by IndoorAtlas [18], a cloud based service that exploits smartphone embedded magnetometer for indoor positioning, while EEG data is acquired by a dedicated hardware composed by a 24-bit analog-to-digital (ADC), a microcontroller and a Bluetooth module. The location and EEG data is then merged using an ad-hoc Android application. The capability to extract valid EEG patterns was tested by projecting visual stimuli on screens along the walking path. Results show that the Evoked Potentials (EP) can be successfully extracted and correlated with position (after 10 seconds of stimulation), with an average error of $2 \mathrm{~m}$. These promising results allow 


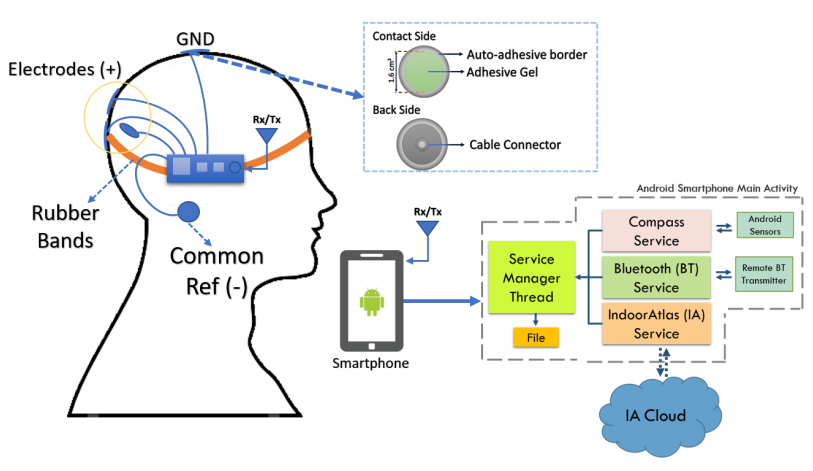

Fig. 1. System Architecture

us to collect an ever-growing dataset to investigate better the interaction between subjects and environments, and to lay the foundation of new paradigms in HMI.

\section{Material And Methods}

In this section we present the system designed to acquire and combine EEG signals and positioning data, along with the analysis performed on the obtained data.

The system is managed by an Android application running on a smartphone that computes the location data and merges it with the EEG raw data, acquired by a dedicated hardware board. The board is composed by a 24-bit analog-to-digital (ADC) converter, a Cortex-M4 microcontroller and a Bluetooth module for communication with the smartphone. The smartphone obtains the positioning data using IndoorAtlas SDK version 2.2.4, with an accuracy of 1 to $3 m$ [18]. After a preliminary setup phase, where the cloud builds a map of the magnetic field of the floorplan (fingerprint), device localization is achieved by streaming to the cloud the magnetometer values.

\section{A. System architecture}

The two main components of the system are the Android application and the EEG acquisition board. The android application acts as master for the system, handling the connections with the EEG acquisition system and the IndoorAtlas cloud, and funnelling all data for synchronization and output file generation.

\section{1) Android Application:}

The Android app architecture is depicted in Fig. 1. An introductory activity welcomes the user and let him/her choose a paired Bluetooth device, then the main activity starts and immediately spawns the ServiceManager thread. The ServiceManager in turn generates three services, each one in charge of collecting a part of the dataset: the Bluetooth Service is designed to connect with the paired Bluetooth device and receive EEG data packets of 11 bytes at 500 $\mathrm{Hz}$; the Compass Service is designed to compute orientation through smartphone magnetometer and accelerometer; the IndoorAtlas Service is designed to receive the device position data from the IndoorAtlas cloud. Each service spawns a dedicated thread for its long-running task, in order not to burden the ServiceManager with high frequency callbacks. Each dedicated thread stores the received data in an internal threadsafe buffer, together with a timestamp. The ServiceManager periodically accesses the three buffers and retrieves data in the current time window using the timestamp information. Then, the ServiceManager performs synchronization of the data received from the three service sources and attaches,

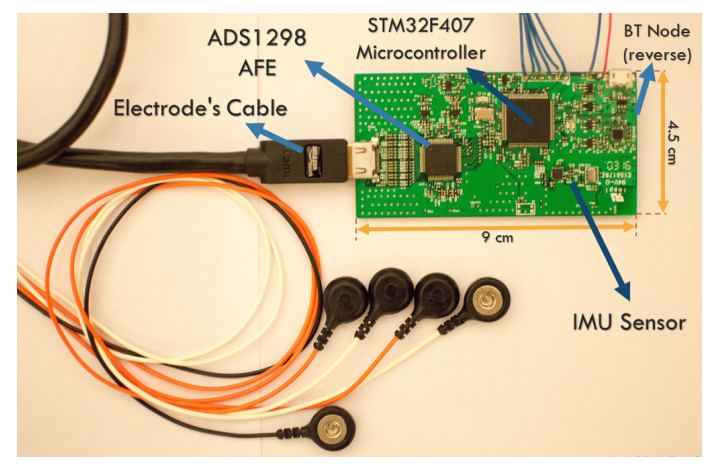

Fig. 2. EEG acquisition board

for each Bluetooth data entry, the respective device position and orientation. Such merged and synchronized data is then stored locally in a file for further offline processing.

\section{2) EEG system Acquisition:}

The EEG signals are acquired with the system implementation presented in Fig. 1[19] [20]. The EEG signals are sampled using the 8-channel Texas Instrument ADS1298 low power analog-to-digital converter, designed for the acquisition of biopotentials (ExG). Each channel has a resolution of 24 bits with a power consumption of $0.75 \mathrm{~mW}$. The sampling rate ranges from $250 \mathrm{~Hz}$ to $32 \mathrm{kHz}$. The current system uses only three channels at $500 \mathrm{~Hz}$, located at $\mathrm{Oz}, \mathrm{Pz}$ and $\mathrm{P} 4$, according to the 10-20 reference system [21], with a common reference to A1 (left earlobe) and GND connected to Fz. Circular gel-based electrodes with a surface contact of $2 \mathrm{~cm}^{2}$ are used to transfer the signals from the skin to the ADC. All the sampled data is then transmitted to a microcontroller via SPI. The STMicroelectronics STM32F407 microcontroller purveys the required computational power. It is based on an ARM Cortex-M4 core running at $168 \mathrm{MHz}$, with floating point unit, $192 \mathrm{kB}$ of SRAM and $1 \mathrm{MB}$ of non volatile Flash memory. Finally, the raw data is transmitted to the Android application using the Bluegiga WT12 Bluetooth Class 2 Module.

These components populate a $9 \times 4.5 \mathrm{~cm} 6$-layer Printed Circuit Board (PCB) as shown in Fig. $2^{1}$. The board's power supply is managed by a dedicated integrated circuit with an internal switching voltage regulator and Low-dropout (LDO) regulators.

\section{B. Signal processing:}

The recorded data is analyzed using initially a timefrequency domain transformation. The spectrogram of the signal is computed to visualize the position/frequency relation over time. For this, the EEG data is down-sampled to $100 \mathrm{~Hz}$. Fig. 3 is obtained using the spectrogram function (Matlab) with window size of 100 samples and 50\% overlap. It is plotted in relation to the cartesian coordinates in $m$ from a corner of the corridor. The points where the position remains unchanged reveal the moments where the subject is receiving the visual stimulation (10 seconds approx). Since the quality of the spectrogram is not sufficient to correctly quantify the EPs, the sections containing the visual stimulation are extracted for a thorough analysis. Given the time-locked nature of the stimuli, signal averaging (on a given time window) is used to increase the signal-to-noise ratio of the

\footnotetext{
${ }^{1}$ The board size and weight can be optimized in a future implementation. Current dimensions furnish reconfiguration flexibility during the tests.
} 


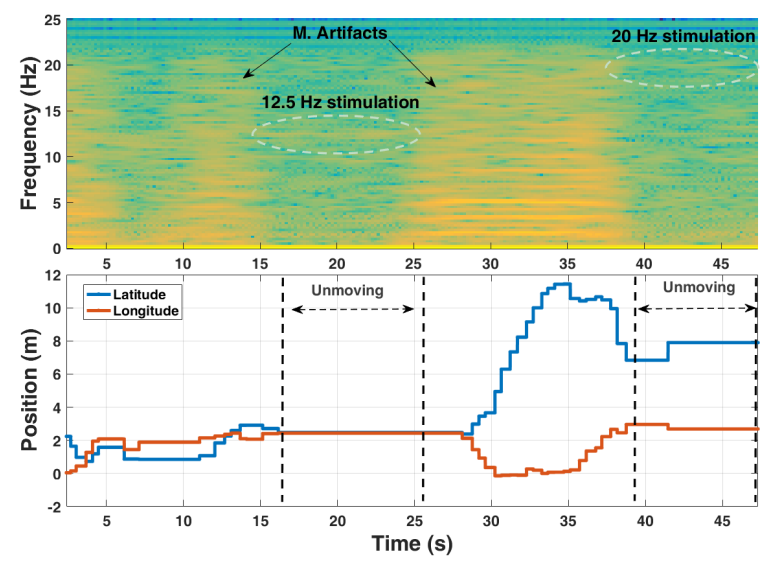

Fig. 3. Spectrogram of the EEG data and positions

segment. Subsequently, the window is analyzed using the frequency tagging technique by average of the frequency components of small chunks of the original signal.

\section{Challenges working with the system.}

The major challenge regarding the Android application is data synchronization between services. In fact, there is no practical way to determine via software the arrival time of a Bluetooth packet with $m s$ precision. The Bluetooth unit of the smartphone stores locally the received packets and delivers them to the Android Bluetooth adapter in batches. The frequency of the batch delivery to the Android Bluetooth adapter depends not only on the remote Bluetooth transmission frequency, but also on the internal implementation of the two units. To overcome this problem, we devised an algorithm to estimate the arrival time of one packet and then we exploit the transmission frequency knowledge to update the timestamp for each following packet. We omit further details of the algorithm for the sake of conciseness.

Regarding the acquisition of the EEG signals, challenges arise because of the different sources of noise. These are normally lessened by carrying out experimentation in carefully controlled environments. Mainly, three sources of interference are present during the recordings. The first corresponds to the movement artifacts (MA), caused by the movement of the electrode and/or the electrical changes due to energy equalization between the subject and the ground. The second, the blink artifacts (BA), normally present in EEG measurements, are generated by the movement of the eyes. The third corresponds to the $50 \mathrm{~Hz}$ power line interference (PLI). While recording on real-life scenarios, the location of the electrodes plays an important role in reducing the effects of the mentioned artifacts, but simultaneously, it can also affect the amplitude of the studied signal. Since SSVEP are mostly present at the right side of the occipital lobe [22]. The use of three electrodes on this region balances complexity and the likelihood of capturing the signal.

The reduction of artifacts is finally achieved after selecting a proper electrode placement. Empirical experimentation was

TABLE I

COMPARISON BETWEEN DIFFERENT ELECTRODE PLACEMENT

\begin{tabular}{|c|c|c|c|c|}
\hline Conf & SNR (SSVEP) & PLI $^{2}$ & BA $^{\mathbf{2}}$ & MA $^{\mathbf{2}}$ \\
\hline $\mathbf{A}$ & 3.14 & 3.26 & 9.29 & 129.25 \\
\hline $\mathbf{B}$ & 4.65 & 2.24 & 39.03 & 130.29 \\
\hline $\mathbf{C}$ & 4.10 & 2.80 & 8.70 & 115.21 \\
\hline
\end{tabular}

${ }^{2}$ Measured in $\mu \mathrm{Vrms}$ performed to quantify the PLI, BA and MA interference in three different configurations. These are: GND and all reference electrodes connected to A1 (A), GND at Fz and all references at connected to the Fp1 (B) and GND at $\mathrm{Fz}$ and all references connected to A1 (C). Table I shows the results of the experimentation, demonstrating that $\mathrm{C}$ rejects successfully more noise than the other configurations, notwithstanding that it does not have the best SNR.

\section{EXPERIMENTAL RESULTS}

The system described in the previous section is tested on one healthy subject with no previous history of neural diseases. The evaluation was performed using the hardwaresoftware implementation presented in section II-A. The board is placed on the subject's head, as shown in 1. The electrodes are located at $\mathrm{Oz}, \mathrm{Pz}$ and $\mathrm{P} 4$, following the 10-20 reference system, while the reference electrode was placed at $\mathbf{F z}$.

The subject was asked to follow the predefined path as indicated in Fig. 4. The visual stimulation is presented along the walking path by two identical Full High-Definition (HD) screens $(15 ", 1920 x 1080 \mathrm{px})$ with a refresh rate $>60$ FPS. The separation between the screen and the user during the stimulation is about 1 meter. The visual stimulation is generated by using a checkerboard (10x10 square elements) covering $35 \%$ of the screen over a grey background. Each screen projected a stimuli with a different frequency. For this experimentation we used frequencies of 12.5 and $20 \mathrm{~Hz}$.

The subject explores the walking path from the starting point. Once the first screen is reached, the subject observes the screen with the corresponding visual stimulation to generate the SSVEP. Subsequently, the user moves to the next screen for the last stimuli observation.

During the task, the subject's position and EEG data is recorded using the implementation presented at the section II-A for posterior offline analysis. The resulting raw data is processed following the methodology presented at II-B. The two plots presented in Fig. 5 and Fig. 6 reveal the capability of the system to detect the generated stimuli. It is worth noticing also that the channel carrying the strongest frequency response is not always the same, specially for different frequencies, which justifies the employment of multiple electrodes.

As an another study case using the proposed platform, we focused on the correlation between the moving artifacts and the displacement of the subject. Fig. 3 shows the frequency components of the artifacts featuring three spectral lines components with a bandwidth of $1 \mathrm{~Hz}$ and a separation of 1.5 $\mathrm{Hz}$ approx. Table II shows the results of experimentation with different increasing walking speeds, where is worth notice that these lines have the tendency to move towards higher frequencies. This characterization may be useful to develop a method to determine the speed of the user or to reduce the effects of these artifacts. This may be covered in a future research.

In summary, the current results show that the Evoked Potentials can be successfully extracted using the proposed system even in a mobile, wearable setup (as opposed to the

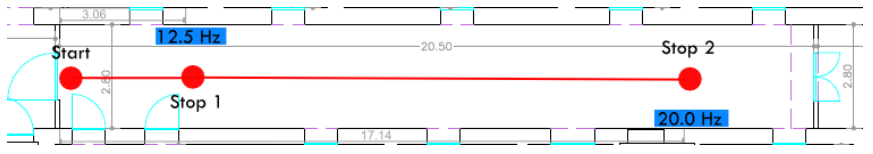

Fig. 4. Predefined path and stimuli location 


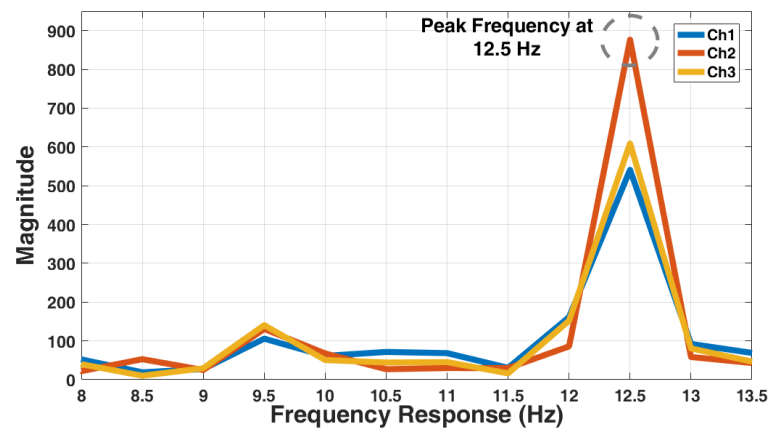

Fig. 5. Frequency Response for SSVEP at $12.5 \mathrm{~Hz}$.

usual stationary conditions used in EEG experiments). This encourage the belief that the current platform will enable us to seek for more complex brain responses in a future research. Also, correlations between indoor position and motion were characterized, which can be used to extract extra information from the signal or to increase the SNR, demonstrating significant opportunity for sensor fusion exploiting real-time location and EEG tracking. It is worth mentioning that all the data-set generated so far and in future experimentations will be publicly available, enabling other researchers to study the brain activity in relation with position. Another important contribution of this work is that all the data is collected in real scenarios. This will challenge the current methods but will empower the development of new algorithms to mitigate the effects of undesired signals present in the environment.

\section{CONCLUSIONS}

In this paper we presented a pilot study in combining EEG signals with subject position data. We described the Hardware-Software platform used for data acquisition and corresponding challenges. We tested the system in a real environment with the experiment described in section III. Experimental results show that it is possible to extract Evoked Potentials from the EEG signal and therefore locate the subject. Also, the detected correlation between EEG artifacts and subject's walking speed encourage us to further investigate over more complex brain responses related to the subject position and motion, since we believe that such research could boost both LBS and BMI.

To allow other researchers to study the brain activity in correlation with indoor positioning, the growing dataset will be publicly available.

\section{ACKNOWLEDGMENT}

The research contribution presented in this paper has been supported by the European project EuroCPS (grant $n$. 644090).

\section{REFERENCES}

[1] J. Kárník and J. Streit, "Summary of available indoor location techniques," IFAC-PapersOnLine, vol. 49, no. 25, pp. 311-317, 2016.

TABLE II

RELATION BETWEEN POSITION AND MA

\begin{tabular}{|c|c|c|c|}
\hline Velocity(Km/h) & CF1 $^{3}$ & CF2 & CF3 \\
\hline $\mathbf{3 . 0 2}$ & 1.4 & 2.8 & 4.3 \\
\hline $\mathbf{3 . 4}$ & 1.5 & 3.1 & 4.7 \\
\hline $\mathbf{4 . 3 5}$ & 1.6 & 3.5 & 5 \\
\hline $\mathbf{4 . 3 5}$ & 1.9 & 3.6 & 5.1 \\
\hline \multicolumn{3}{|c}{ Central Frequency (CFx) measured in $\mathrm{Hz}$} \\
\hline
\end{tabular}

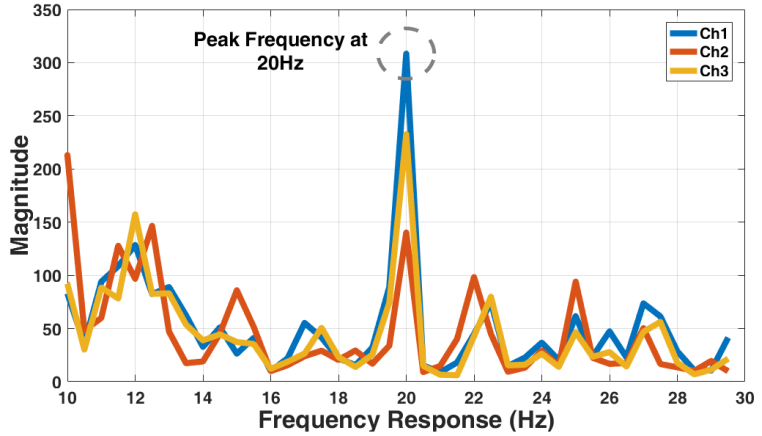

Fig. 6. Frequency Response for SSVEP at $20 \mathrm{~Hz}$.

[2] C. Yang and H.-R. Shao, "Wifi-based indoor positioning," IEEE Communications Magazine, vol. 53, no. 3, pp. 150-157, 2015.

[3] D. A. G. L. S. Moghtadaiee, Vahideh, "Indoor localization using fm radio signals: A fingerprinting approach," in Indoor Positioning and Indoor Navigation (IPIN). IEEE, 2011.

[4] J. Larranaga, L. Muguira, J.-M. Lopez-Garde, and J.-I. Vazquez, "An environment adaptive zigbee-based indoor positioning algorithm," in Indoor Positioning and Indoor Navigation (IPIN), 2010 International Conference on. IEEE, 2010, pp. 1-8.

[5] S. Liu, Y. Jiang, and A. Striegel, "Face-to-face proximity estimationusing bluetooth on smartphones," IEEE Transactions on Mobile Computing, vol. 13, no. 4, pp. 811-823, April 2014.

[6] Y. Chu and A. Ganz, "A uwb-based 3d location system for indoor environments," in Broadband Networks, 2005. BroadNets 2005. 2nd International Conference on. IEEE, 2005, pp. 1147-1155.

[7] L. M. Ni, Y. Liu, Y. C. Lau, and A. P. Patil, "Landmarc: indoor location sensing using active rfid," in Proceedings of the First IEEE International Conference on Pervasive Computing and Communications, 2003. (PerCom 2003)., March 2003, pp. 407-415.

[8] J. Chung, M. Donahoe, C. Schmandt, I.-J. Kim, P. Razavai, and M. Wiseman, "Indoor location sensing using geo-magnetism," in Proceedings of the 9th international conference on Mobile systems, applications, and services. ACM, 2011, pp. 141-154.

[9] Q. Lu, X. Liao, S. Xu, and W. Zhu, "A hybrid indoor positioning algorithm based on wifi fingerprinting and pedestrian dead reckoning," in Personal, Indoor, and Mobile Radio Communications (PIMRC), 2016 IEEE 27th Annual International Symposium on. IEEE, 2016.

[10] C.-C. Chiu, J.-C. Hsu, and J.-S. Leu, "Implementation and analysis of hybrid wireless indoor positioning with ibeacon and wi-fi," in Ultra Modern Telecommunications and Control Systems and Workshops (ICUMT), 2016 8th International Congress on. IEEE, 2016.

[11] J. Park, I. Woo, and S. Park, "Application of eeg for multimodal human-machine interface," in 2012 12th International Conference on Control, Automation and Systems, Oct 2012, pp. 1869-1873.

[12] M. Münch, G. Plomp, E. Thunell, A. Kawasaki, J. Scartezzini, and M. Herzog, "Different colors of light lead to different adaptation and activation as determined by high-density eeg," Neurolmage, 2014.

[13] C. M. Hagerhall, T. Laike, R. P. Taylor, M. Küller, R. Küller, and T. P. Martin, "Investigations of human eeg response to viewing fractal patterns," Perception, vol. 37, no. 10, pp. 1488-1494, 2008.

[14] O. Sourina, Y. Liu, and M. K. Nguyen, "Emotion-enabled eeg-based interaction," in SIGGRAPH Asia 2011 Posters, 2011.

[15] A. Turnip, D. Soetraprawata, and T. A. Tamba, "Eeg-ssvep signals extraction with nonlinear adaptive filter for brain-controlled wheelchair," in International Conference on Control, Automation and Systems, 2015.

[16] B. Rebsamen, E. Burdet, C. Guan, H. Zhang, C. L. Teo, Q. Zeng, C. Laugier, and M. H. A. Jr., "Controlling a wheelchair indoors using thought," IEEE Intelligent Systems, vol. 22, no. 2, March 2007.

[17] M. S. Hossain, "Cloud-supported cyber-physical localization framework for patients monitoring," IEEE Systems Journal, 2015.

[18] IndoorAtlas, "Homepage," https : / / www. indooratlas.com/.

[19] M. Tomasini, S. Benatti, B. Milosevic, E. Farella, and L. Benini, "Power line interference removal for high-quality continuous biosignal monitoring with low-power wearable devices," IEEE Journal, 2016.

[20] V. Kartsch, S. Benatti, D. Rossi, and L. Benini, "A wearable eegbased drowsiness detection system with blink duration and alpha waves analysis," in 8th International IEEE EMBS Conference on Neural Engineering. IEEE, 2017.

[21] R. W. Homan, J. Herman, and P. Purdy, "Cerebral location of international 10-20 system electrode placement," Electroencephalography and Clinical Neurophysiology, vol. 66, no. 4, pp. $376-382,1987$.

[22] Y. Wang, Z. Zhang, X. Gao, and S. Gao, "Lead selection for ssvepbased brain-computer interface," in Engineering in Medicine and Biology Society, 2004. IEMBS'04. IEEE. 